Article

\title{
Compatibility of maximum inorganic and organic calcium and phosphate content in neonatal parenteral solutions
}

\author{
Dorota Watrobska-Swietlikowska \\ Department of Pharmaceutical Technology, Medical University of Gdansk, Hallera Av. 107, 80-416 Gdansk, \\ Poland, email: dwatro@gumed.edu.pl \\ * Correspondence: dwatro@gumed.edu.pl; Tel.: +48-58-349-10-85
}

\begin{abstract}
The aim of the study was to determine the maximum safe concentration of calcium and phosphate in neonatal parenteral nutrition $(\mathrm{PN})$ solutions when various combinations of inorganic and organic salts are applied. Twelve PN solutions for neonatal use were aseptically prepared. Increasing concentration of inorganic and organic calcium and phosphate were added to the standard formulas. Each admixture was separately tested according to following conditions; after mixing, $37^{\circ} \mathrm{C}$ for $24 \mathrm{~h}$, and maximum safe combination of calcium and phosphate were stored at $4^{\circ} \mathrm{C}$ for 30 days and followed by $24 \mathrm{~h}$ at $37^{\circ} \mathrm{C}$. Visual inspections against a black and white contrast background, microscopic observation of undiluted PN solutions as well as the membrane filter after filtration of the PN solution, $\mathrm{pH}$ evaluation, and spectrophotometry at $600 \mathrm{~nm}$ were examined in triplicate. Safe maximum concentration of organic and inorganic calcium and phosphate was proposed individually for each composition of parenteral nutrition solutions. Surprisingly organic calcium with organic phosphate showed precipitation but over the therapeutic range. The protective effect of amino acid was observed and higher concentrations of calcium and phosphate were free of precipitation.
\end{abstract}

Keywords: parenteral nutrition, neonatal solution; calcium; phosphate; organic; inorganic; precipitation;

\section{Introduction}

Neonatal and paediatric parenteral nutrition admixtures $(\mathrm{PN})$ are very complex and difficult due to the small volume and numerous additives, making them highly concentrated solutions. The most important physical incompatibility in parenteral nutrition $(\mathrm{PN})$ is precipitation of calcium phosphate, particularly for preterm infants who require high concentrations of these electrolytes in a small volume [1]. The insoluble precipitate may result in serious clinical complications such as catheter occlusion or even microvascular pulmonary emboli [1].

In parenteral nutrition, organic (calcium gluconate) and inorganic (calcium chloride) calcium salts are available. The chloride salt contains a higher proportion of calcium available to associate with free phosphate, leading to precipitation due to calcium chloride's larger dissociation constant in comparison to calcium gluconate [2]. Furthermore, organic calcium salts have a lower degree of dissociation compared to inorganic calcium chloride [3]. These reason cause that gluconate salt should be the predominant calcium source in compounding PN and it is only recommended in nutrition for preterm infants and children. In fact, there is a recommendation that an inorganic calcium salt such as calcium chloride should never be used in parenteral nutrition in children and in adults as well [4]. But there are problems connected with using organic calcium salt. On the one hand, due to better its precipitation profile, organic calcium salt (calcium gluconate) is preferred in 
compounding parenteral admixtures, however many authors suggest that it pollutes parenteral admixtures with aluminium [5,6]. So, to minimise the aluminium content in PN admixtures, inorganic calcium salt is preferred.

Phosphate salts are inorganic (sodium phosphate) as well as organic (sodium glycerophosphate, NaGP) salts. Phosphate salts are supplied as a concentrated solution, NaGP contains $1 \mathrm{mmol} / \mathrm{mL}$ of phosphate whereas inorganic ones contain $2 \mathrm{mmol} / \mathrm{mL}$ of phosphate. NaGP is covalently bound to a glycerol backbone which hinders the formation of a precipitate with divalent calcium ions. The manufacturer reports the compound is stable in solutions compounded at concentrations up to $120 \mathrm{mmol} / \mathrm{L} \mathrm{NaGP}$ (organic salt) and $96 \mathrm{mEq} / \mathrm{L}$ calcium chloride. The dibasic calcium phosphate salt is 60 times less soluble than the monobasic form in aqueous medium and its solubility is highly pH-dependant [7]. Furthermore, the use of organic phosphates considerably increases the Ca-P compatibility. It was confirmed that the organic phosphate has superior stability in comparison to dibasic sodium phosphate over a range of $\mathrm{pH}$, temperature, calcium used and amino acid or glucose concentrations [3,8].

Many authors try to determine the maximum concentrations of calcium and phosphate at various combinations that can be mixed safely, but it cannot be universally determined due to the variety of composition of compounding products $[1,2,3,6,9]$. Precipitation of calcium phosphate is much more frequent and dangerous in small patients (premature, newborn and children) because the precipitation is more likely to occur at lower volumes and, moreover, is an endothermic reaction (incubators used for neonates increase the likelihood of precipitation). In fact, the increase of temperature will have two effects. One is the dissociation of calcium from its organic form; this will increase the availability of free calcium ions which will react with phosphate. Secondly, raising the temperature of a mixture may also shift the phosphate equilibrium from a mono- to a dibasic salt. However some date confirm that NaGP and calcium chloride compatibility provides a clinical option for limiting aluminium contamination while providing sufficient calcium and phosphate to meet the needs of neonatal patients [10].

It is important to know that unaided visual observation of precipitation is limited to approximately $50 \mu \mathrm{m}$ individual particles and can be highly variable. Subvisible precipitates ranging from 5 to $50 \mu \mathrm{m}$ may occlude the microvasculature, such as in the pulmonary system. Particles below $5 \mu \mathrm{m}$, especially lipid globules, clog the pulmonary capillaries, producing an embolic syndrome and causing cell death mainly in pre- term infants [11]. Studies employing particle detection and size measurement by light obscuration provide objective evidence of subvisible microprecipitation which can be clinically dangerous [12]. Understanding the chemical and practical compatibility of calcium phosphate salts injections is critical to ensuring the safe i.v. administration of these supplements and preventing patient harm.

The aim of the study was to assess the risk of precipitation calcium phosphate when vary concentrations of calcium and phosphate salts inorganic and organic are mixed in various combinations in standard paediatric PN solutions. Our study assists practitioners in preventing calcium and phosphate precipitates in parenteral solutions.

\section{Materials and Methods}

\subsection{Composition and preparation of PN solutions}

Twelve basic (without calcium and phosphate salts) PN solutions were prepared aseptically following international recommendations under a laminar airflow hood in a class 
A horizontal laminar-airflow hood, located in a GMP class B clean room at the Stanley Dudrick's Memorial Hospital in Skawina, Poland. Single-chamber, monolayer ethylenovinylacetate bags, Exacta-Mix Eva Bag Parenteral, constituting the packaging of PN solutions was used. For this purpose, a Baxa 24 computer-controlled mixer was applied, allowing for precise transfusion following base fluids, i.e., 40\% dextrose solution (B. Braun Melsungen, Germany), amino acids solution (Aminoven Infant $10 \%$ or Vaminolact, Fresenius Kabi, Uppsala, Sweden or Primene 10\%, Baxter Dutschland GMBH, Germany), water for injections, sodium chloride (Natrium chloratum 10\%, Polpharma, Starogard Gdanski, Poland), potassium chloride solution (Kalium chloratum 15\%, WZF Polfa, Warsaw, Poland), magnesium sulphate solution (Magnesii sulfuricum 20\%, Polpharma, Starogard Gdanski, Poland) and trace elements (Peditrace, Fresenius Kabi, Uppsala, Sweden). No lipids were added because they would obscure the presence of a precipitate. All products in this study were approved for use in Poland for parenteral nutrition. Each of the twelve of PN solutions was prepared triplicate and was tested for compatibility using various concentrations of amino acid solutions and glucose (Table 1).

Table 1. Composition of PN solutions under test.

\begin{tabular}{ccccccccc}
\hline $\begin{array}{c}\text { PN } \\
\text { solu } \\
\text { tion }\end{array}$ & $\begin{array}{c}\text { Amino acids } \\
\text { solution }\end{array}$ & $\begin{array}{c}\text { Amino } \\
\mathbf{a c i d} \\
(\mathbf{g} / \mathbf{L})\end{array}$ & $\begin{array}{c}\text { Glucose } \\
(\mathbf{g} / \mathbf{L})\end{array}$ & $\begin{array}{c}\mathbf{N a}^{+} \\
(\mathbf{m m o l} / \mathbf{L})\end{array}$ & $\begin{array}{c}\mathbf{K}^{+} \\
(\mathbf{m m o l} / \mathbf{L})\end{array}$ & $\begin{array}{c}\mathbf{M g}^{2+} \\
(\mathbf{m m o l} / \mathbf{L})\end{array}$ & $\begin{array}{c}\text { Peditrace } \\
(\mathbf{m l} / \mathbf{L})\end{array}$ & $\begin{array}{c}\text { Total } \\
\text { volume } \\
(\mathbf{m l})\end{array}$ \\
\hline P1 & Primene 10\% & 10 & 50 & 200 & 100 & 10 & 15 & 500 \\
P2 & Primene 10\% & 10 & 200 & 200 & 100 & 10 & 15 & 500 \\
P3 & Primene 10\% & 40 & 50 & 200 & 100 & 10 & 15 & 500 \\
P4 & Primene 10\% & 40 & 200 & 200 & 100 & 10 & 15 & 500 \\
A1 & Aminoven 10\% & 10 & 200 & 200 & 100 & 10 & 15 & 500 \\
A2 & Aminoven 10\% & 10 & 50 & 200 & 100 & 10 & 15 & 500 \\
A3 & Aminoven 10\% & 40 & 50 & 200 & 100 & 10 & 15 & 500 \\
A4 & Aminoven 10\% & 40 & 200 & 200 & 100 & 10 & 15 & 500 \\
V1 & Vaminolact & 10 & 50 & 200 & 100 & 10 & 15 & 500 \\
V2 & Vaminolact & 10 & 200 & 200 & 100 & 10 & 15 & 500 \\
V3 & Vaminolact & 40 & 50 & 200 & 100 & 10 & 15 & 500 \\
V4 & Vaminolact & 40 & 100 & 180 & 100 & 10 & 15 & 500 \\
\hline
\end{tabular}

\subsection{Storage and sampling}

To analyse the compatibility of calcium and phosphate, in the first step $100 \mathrm{~mL}$ of each basic PN solution was transferred to glass bottles, increasing organic or inorganic calcium (Calcium gluconate solution 10\%, Monico SPA, Mestre, Venezia and Calcii chloratum 10\%, Polfa Warszawa, Warsow, Poland, respectively) and phosphate (Glycophos, Fresenius Kabi, Uppsala, Sweden and Addiphos, Fresenius Kabi, Uppsala, Sweden, respectively) concentration above therapeutic use were added. 
Each of the $12 \mathrm{PN}$ solutions with various combinations of calcium and phosphate was tested in triplicate. All samples with increasing concentration of calcium and phosphate ions (the procedure of preparing the samples with increasing concentration of these ions is described in 2.4. Data interpretation) were stored in glass bottles for 24 hours at $37^{\circ} \mathrm{C}$ prior to analysis. Solutions were placed in a thermostatic chamber at $37^{\circ} \mathrm{C}$ (without any light) for 24 hours to simulate paediatric conditions similar to body temperature. In the second step, the samples with the maximum safe (that is mean without any precipitation after $24 \mathrm{~h}$ ) concentration of calcium were stored in monolayer ethylenovinylacetate bags (the same used for preparing PN solutions) for 30 days at $4^{\circ} \mathrm{C}$ followed by $24 \mathrm{~h}$ at $37^{\circ} \mathrm{C}$ under regular light conditions, without any light protection. At the beginning $(\mathrm{t}=0)$ and at the end $(t=31)$ of the storage period, physical analyses were performed.

\subsection{Evaluation of Physicochemical Stability}

The physical analyses were performed at $\mathrm{t}=0$ and $24 \mathrm{~h}$ (samples with increasing calcium and phosphate concentration) and at $\mathrm{t}=0$ and 30 days $+24 \mathrm{~h}$ (samples with the maximum safe calcium and phosphate concentration determined in the first step of the study, after $24 \mathrm{~h}$ ). The study was performed with three different aliquots of each PN solution under test. To guarantee the homogenisation of solutions after storage for $24 \mathrm{~h}$ as well as 30 days $+24 \mathrm{~h}$, the samples were gently agitated before analysis.

\subsubsection{Visual observation}

The samples were visually inspected against a black and white contrast background for evidence of precipitation.

\subsubsection{Microscopic Observation}

Each PN solution with increasing concentration of calcium and phosphate was checked microscopically by a 100× light optic microscope with camera (B1 223A Motic, Wetzlar, Germany) for microcrystals. Using recommendations of European Pharmacopoeia (Ph Eur) for particle count, solutions were considered physically compatible if the crystal count was less than 12 particles per $\mathrm{mL}$ measuring $\geq 10 \mu \mathrm{m}$ and no more than 2 particles per $\mathrm{mL}$ measuring $\geq 25 \mu \mathrm{m}$ in diameter. $100 \mathrm{~mL}$ of each of the PN solutions with the maximum safe concentration of calcium and phosphate salts in different combinations were infused through a 0.45-micron nitrocellulose filter disk (Millipore, Billerica, MA) were evaluated for crystal content. Each disk was analysed quantitatively for crystal precipitates under 100× magnification using a linear scale. Physically compatible solutions could contain no more than 2400 particles measuring $\geq 10 \mu \mathrm{m}$ in diameter and 400 particles measuring $\geq 25$ $\mu \mathrm{m}$ in diameter.

\subsection{3. $p H$ Measurement}

The $\mathrm{pH}$ values of the PN solutions were determined at $25^{\circ} \mathrm{C}$ ( $\mathrm{pH}$-Meter type 350, Orion-Research, Boston, USA with combined electrode (Type ERH-11, Hydromet, Warsaw, Poland). Before each pH measurement, a two-point calibration of the $\mathrm{pH}$ meter was done, each with buffer solutions of $\mathrm{pH}$ 7.00 and $\mathrm{pH} 4.00$, respectively. The $\mathrm{pH} 7.00$ solution was used afterwards as a control. Between the calibration steps, the electrode was rinsed with distilled water and wiped dry. Each sample was triplicate measured after 3 min of equilibration. 


\subsubsection{Light scattering}

PN solutions were examined for light scattering by a UV spectrophotometer JASCO V-530 (Jasco International CO. LTD., Tokio, Japan) at $600 \mathrm{~nm}$ against sterile water for injection blank. An absorbance of greater than 0.06 was determined as the "arbitrary threshold for precipitation".

\subsection{Data interpretation}

Results were classified in three categories: "stability zone" for clear samples without precipitation, "high risk zone" for areas of concentration without measurements and "precipitation zone" when visible particles or precipitates were detected. "High risk zone" was the zone without measurements. Increasing step by step quantities of calcium and phosphate in the four different combinations (inorganic and organic salts) in concentrations ranging from 1 to $110 \mathrm{mmol} / \mathrm{L}$ depending on the composition of the basic PN solution were used to establish calcium phosphate precipitation zones.

When inorganic salts were used, concentrations were increased in steps of $1 \mathrm{mmol} / \mathrm{L}$ for each salt (Ca and $\left.\mathrm{PO}_{4}\right)$, beginning at $1 \mathrm{mmol} / \mathrm{L}$ and continuing to $30 \mathrm{mmol} / \mathrm{L}$, then $10 \mathrm{mmol} / \mathrm{L}$ were added to refine the curve to $50 \mathrm{mmol} / \mathrm{L}$. When organic salts were used, the steps between the two concentrations were $10 \mathrm{mmol} / \mathrm{L}$ of each salt, beginning at $10 \mathrm{mmol} / \mathrm{L}$ and continuing, depending on composition, to 50 or even $110 \mathrm{mmol} / \mathrm{L}$.

\subsection{Statistical analysis}

The results are presented as mean and standard deviation (SD). Across at ime points, the results were evaluated using the non-parametric ANOVA Friedman test. Statistica 13 software (StatSoft, Kraków, Poland) was used. The priori level of significance was 0.05 .

\section{Results}

\subsection{Visual inspection}

In PN solutions P1, P2, P3, P4, A3, V2 and V3 the maximum concentration of organic ions of calcium and phosphate that did not cause opalescence after $24 \mathrm{~h}$ at $37^{\circ} \mathrm{C}$ was $80 \mathrm{mmol} / \mathrm{L}$ and $60 \mathrm{mmol} / \mathrm{L}$, respectively (Figure 1). For PN solutions $\mathrm{A} 4$ and $\mathrm{V} 4$, the highest concentrations of organic salts were $90 \mathrm{mmol} / \mathrm{L}$ of calcium and $70 \mathrm{mmol} / \mathrm{L}$ of phosphate and for A1, A2, V1 were $70 \mathrm{mmol} / \mathrm{L}$ and 50 $\mathrm{mmol} / \mathrm{L}$, respectively. Despite the fact that calcium and phosphate were organic salts, turbidity was observed but above the therapeutic range (Figure 1, Figure 2).

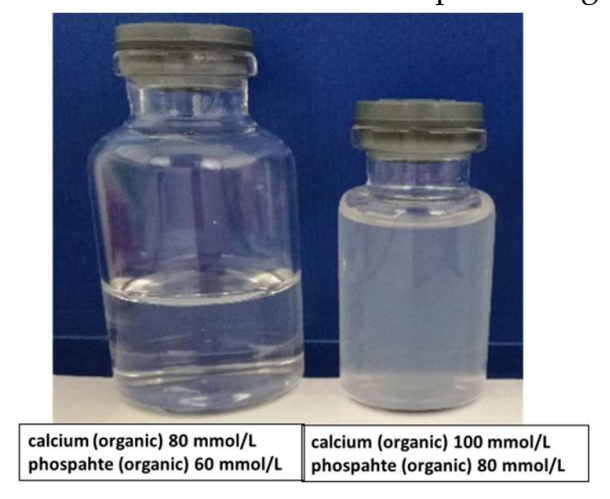

Fig. 1. PN solution P2 with increasing the concentration of organic salts - calcium and phosphate $\left(\mathrm{t}=24 \mathrm{~h}, 37^{\circ} \mathrm{C}\right)$. 

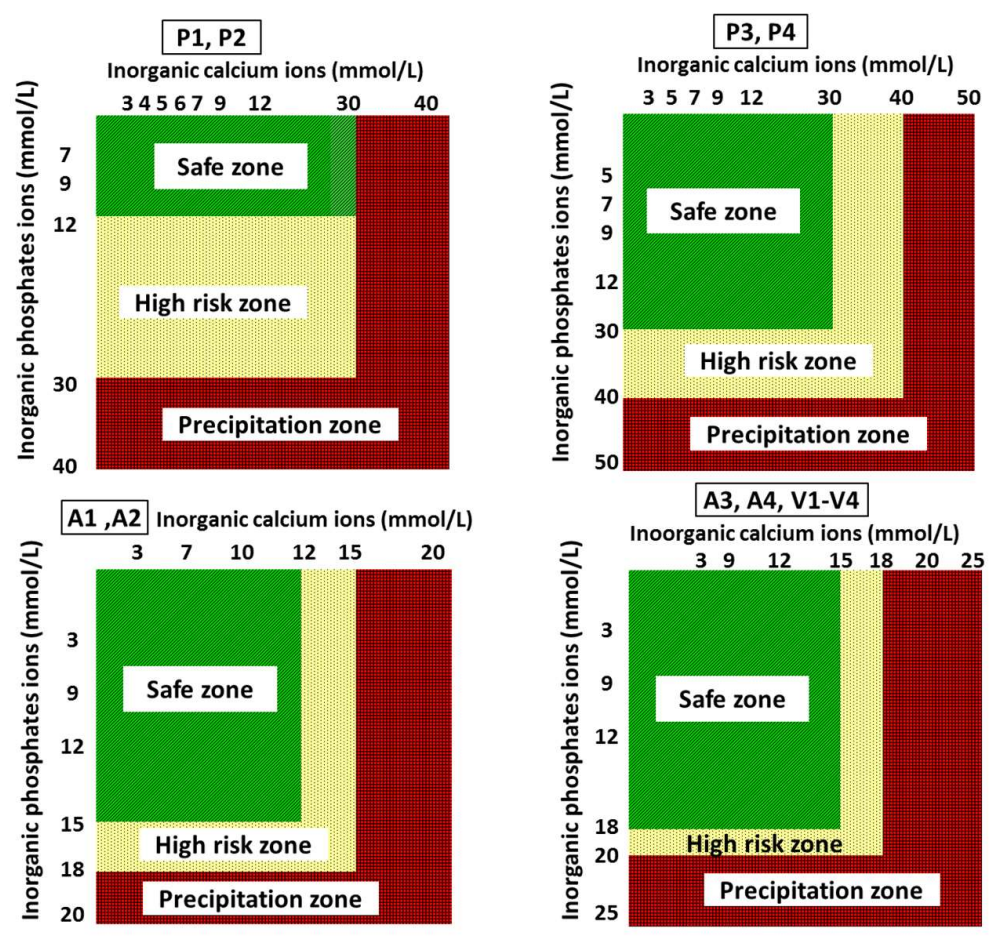

Fig. 2. Stability and precipitation zones of PN solution in respect of the concentration of organic and inorganic calcium and organic phosphate ions $\left(\mathrm{t}=24 \mathrm{~h}, 37^{\circ} \mathrm{C}\right)$.

Smaller concentration of organic calcium and inorganic phosphate showed precipitation (Figure 3). In PN solutions P3, P4 the maximum concentration of organic calcium and inorganic phosphate that did not cause opalescence after $24 \mathrm{~h}$ at $37^{\circ} \mathrm{C}$ was $30 \mathrm{mmol} / \mathrm{L}$ and $30 \mathrm{mmol} / \mathrm{L}$, respectively, whereas in PN solutions P1 and P2 (smaller content of glucose and amino acids) was only $30 \mathrm{mmol} / \mathrm{L}$ and $12 \mathrm{mmol} / \mathrm{L}$ (Figure 3). Using Aminoven or Vaminolact amino acids solutions allowed to combine much smaller content of calcium and phosphate (Figure 3). The same dependences were noticed when inorganic calcium and inorganic phosphate was used (Figure 4). 

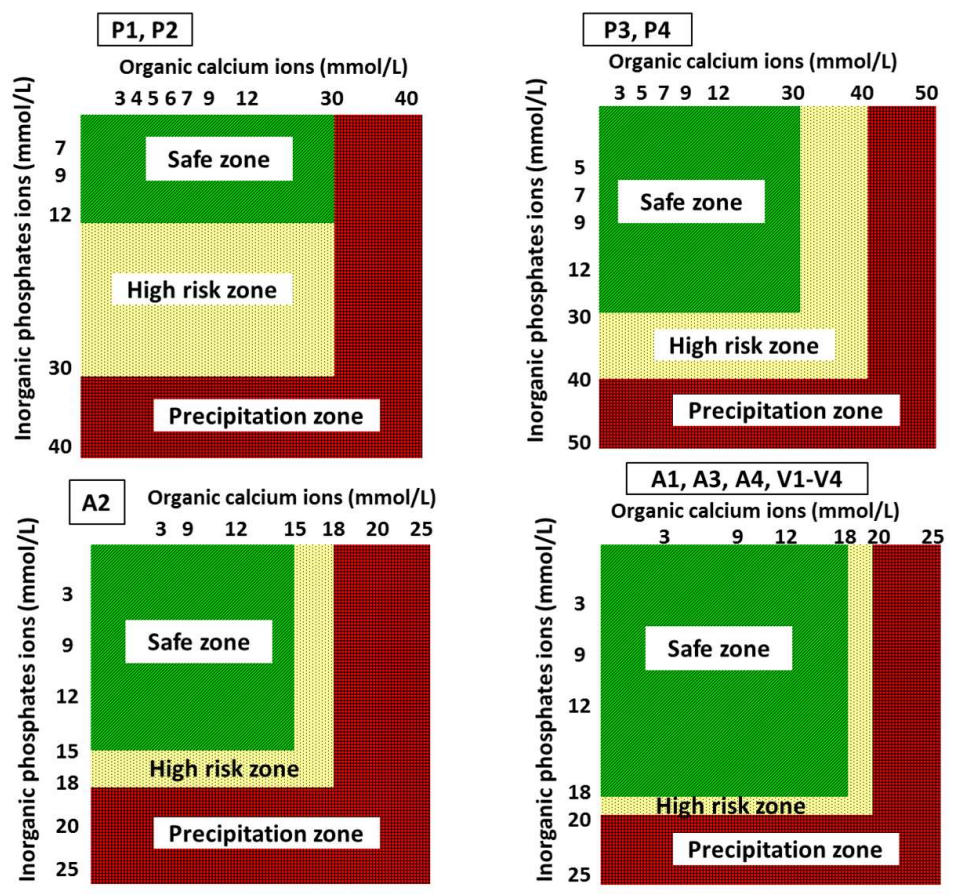

Fig. 3. Stability and precipitation zones of PN solution in respect of the concentration of organic calcium and inorganic phosphate ions $\left(\mathrm{t}=24 \mathrm{~h}, 37^{\circ} \mathrm{C}\right)$.
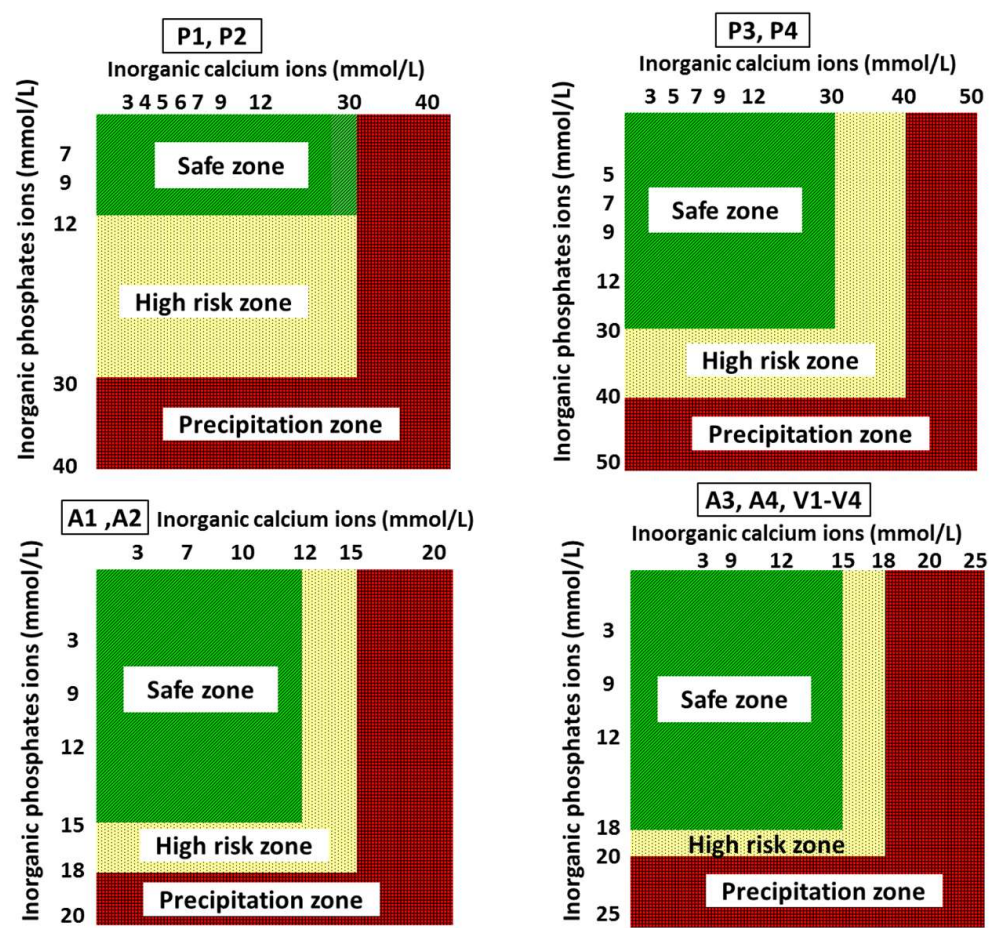

Fig. 4. Stability and precipitation zones of PN solution in respect of the concentration of inorganic calcium and inorganic phosphate ions $\left(\mathrm{t}=24 \mathrm{~h}, 37^{\circ} \mathrm{C}\right)$.

After 30 days $+24 \mathrm{~h}$ of storage, no changes were observed in the presence of organic calcium and organic phosphate ions in all the aqueous phases with the maximum safe (without precipitation after $24 \mathrm{~h}$ ) ion concentration, regardless of the amino acid preparation and composition (Table 2-4). 
Other combinations of calcium and phosphate ions in therapeutic range showed a characteristic white crystalline precipitate, falling to the bottom (Table 2-4). This concerned the PN solutions P1, V1 in which inorganic calcium and phosphate ions were combined, as well as P1, P2, P3, P4, A1, A3, A4 and V2 with organic calcium and inorganic phosphate ions. When mixing safe inorganic calcium salt with inorganic phosphate nearly all PN solutions (except A1, V2 and V4) showed precipitation after 30 days $+24 \mathrm{~h}$ of storage.

Limitation: Analysis of samples with precipitation after 31 days of storage should be done more frequently from $\mathrm{t}=0$ to $\mathrm{t}=31$ days to determine the point when precipitation occurred.

Table 2. Physicochemical properties of PN solution composition $\mathrm{P}(\mathrm{n}=9$; mean $\pm \mathrm{SD}$; $\mathrm{p}<0.05$, between 0 and 30 days +24 hours)

\begin{tabular}{|c|c|c|c|c|c|c|c|}
\hline $\begin{array}{c}\text { PN } \\
\text { solution } \\
\end{array}$ & $\begin{array}{l}\text { Calcium } \\
{[\mathrm{mmol} / \mathrm{l}]} \\
\end{array}$ & $\begin{array}{c}\text { Phosphate } \\
{[\mathrm{mmol} / \mathrm{l})}\end{array}$ & $\begin{array}{c}\text { Time } \\
\text { [days] }\end{array}$ & $\begin{array}{c}\text { Visual } \\
\text { observation } \\
\end{array}$ & Absorbance & $\begin{array}{l}\text { Microscopic } \\
\text { observation }\end{array}$ & $\mathrm{pH}$ \\
\hline \multirow{8}{*}{$\mathrm{P} 1$} & \multirow{2}{*}{ org 80} & \multirow{2}{*}{ org 60} & 0 & $x$ & $0.0019 \pm 0.0001$ & $x$ & $7.18 \pm 0.01$ \\
\hline & & & 31 & $x$ & $0.0018 \pm 0.0002$ & $x$ & $6.82 \pm 0.00$ \\
\hline & \multirow{2}{*}{ org 30} & \multirow{2}{*}{ inorg 12} & 0 & $x$ & $0.0034 \pm 0.0001$ & $x$ & $5.82 \pm 0.02$ \\
\hline & & & 31 & $\mathrm{~V}$ & $0.1134 \pm 0.004$ & $\mathrm{~V}$ & $5.93 \pm 0.01$ \\
\hline & \multirow{2}{*}{ inorg 30} & \multirow{2}{*}{ org 30} & 0 & $x$ & $0.0037 \pm 0.0001$ & $x$ & $6.37 \pm 0.00$ \\
\hline & & & 31 & $x$ & $0.0030 \pm 0.0001$ & $x$ & $6.44 \pm 0.01$ \\
\hline & \multirow{2}{*}{ inorg 30} & \multirow{2}{*}{ inorg 12} & 0 & $x$ & $0.0052 \pm 0.0002$ & $x$ & $5.86 \pm 0.02$ \\
\hline & & & 31 & $\mathrm{~V}$ & $0.1224 \pm 0.008$ & $\mathrm{~V}$ & $5.41 \pm 0.01$ \\
\hline \multirow{8}{*}{ P2 } & \multirow{2}{*}{ org 80} & \multirow{2}{*}{ org 60} & 0 & $x$ & $0.0014 \pm 0.0001$ & $x$ & $7.01 \pm 0.02$ \\
\hline & & & 31 & $x$ & $0.0014 \pm 0.0002$ & $x$ & $7.01 \pm 0.01$ \\
\hline & \multirow{2}{*}{ org 30} & \multirow{2}{*}{ inorg 12} & 0 & $x$ & $0.0023 \pm 0.0001$ & $x$ & $5.63 \pm 0.01$ \\
\hline & & & 31 & $x$ & $0.0017 \pm 0.0001$ & $x$ & $5.82 \pm 0.02$ \\
\hline & \multirow{2}{*}{ inorg 30} & \multirow{2}{*}{ org 30} & 0 & $x$ & $0.0014 \pm 0.0001$ & $x$ & $5.95 \pm 0.00$ \\
\hline & & & 31 & $x$ & $0.0017 \pm 0.0002$ & $x$ & $6.29 \pm 0.00$ \\
\hline & \multirow{2}{*}{ inorg 30} & \multirow{2}{*}{ inorg 12} & 0 & $x$ & $0.0012 \pm 0.0001$ & $x$ & $5.52 \pm 0.01$ \\
\hline & & & 31 & $\mathrm{~V}$ & $0.2131 \pm 0.007$ & $\mathrm{~V}$ & $5.54 \pm 0.01$ \\
\hline \multirow{8}{*}{ P3 } & \multirow{2}{*}{ org 80} & \multirow{2}{*}{ org 60} & 0 & $x$ & $0.0014 \pm 0.0001$ & $X$ & $6.84 \pm 0.02$ \\
\hline & & & 31 & $x$ & $0.0025 \pm 0.0001$ & $x$ & $6.49 \pm 0.02$ \\
\hline & \multirow{2}{*}{ org 30} & \multirow{2}{*}{ inorg 30} & 0 & $x$ & $0.0081 \pm 0.0003$ & $x$ & $5.53 \pm 0.01$ \\
\hline & & & 31 & $\mathrm{~V}$ & $0.1711 \pm 0.009$ & $\mathrm{~V}$ & $5.58 \pm 0.02$ \\
\hline & \multirow{2}{*}{ inorg 30} & \multirow{2}{*}{ org 30} & 0 & $x$ & $0.0015 \pm 0.0001$ & $X$ & $5.78 \pm 0.01$ \\
\hline & & & 31 & $x$ & $0.0031 \pm 0.0001$ & $X$ & $6.00 \pm 0.01$ \\
\hline & \multirow{2}{*}{ inorg 30} & \multirow{2}{*}{ inorg 30} & 0 & $x$ & $0.0009 \pm 0.0001$ & $x$ & $5.41 \pm 0.00$ \\
\hline & & & 31 & $\mathrm{~V}$ & $0.2155 \pm 0.008$ & $\mathrm{~V}$ & $5.24 \pm 0.01$ \\
\hline \multirow{2}{*}{$\mathrm{P} 4$} & \multirow{2}{*}{ org 80} & \multirow{2}{*}{ org 60} & 0 & $x$ & $0.0009 \pm 0.0001$ & $x$ & $6.79 \pm 0.01$ \\
\hline & & & 31 & $x$ & $0.0020 \pm 0.0002$ & $x$ & $6.43 \pm 0.01$ \\
\hline
\end{tabular}




\begin{tabular}{|c|c|c|c|c|c|c|}
\hline \multirow{2}{*}{ org 30} & \multirow{2}{*}{ inorg 30} & 0 & $x$ & $0.0005 \pm 0.0001$ & $x$ & $5.68 \pm 0.00$ \\
\hline & & 31 & V & $0.2088 \pm 0.008$ & V & $5.51 \pm 0.02$ \\
\hline \multirow{2}{*}{ inorg 30} & \multirow{2}{*}{ org 30} & 0 & $x$ & $0.0009 \pm 0.0001$ & $x$ & $5.82 \pm 0.01$ \\
\hline & & 31 & $x$ & $0.0013 \pm 0.0001$ & $x$ & $5.94 \pm 0.01$ \\
\hline \multirow{2}{*}{ inorg 30} & \multirow{2}{*}{ inorg 30} & 0 & $x$ & $0.0006 \pm 0.0001$ & $x$ & $5.57 \pm 0.01$ \\
\hline & & 31 & $\mathrm{~V}$ & $0.1899 \pm 0.008$ & $\mathrm{~V}$ & $5.23 \pm 0.01$ \\
\hline
\end{tabular}

$\mathrm{X}=$ no precipitation $\quad \mathrm{V}=$ precipitation

Table 3. Physicochemical properties of PN solution composition A $(n=9 ;$ mean \pm SD; $p<0.05$, between 0 and 30 days +24 hours)

\begin{tabular}{|c|c|c|c|c|c|c|c|}
\hline $\begin{array}{c}\text { PN } \\
\text { solution }\end{array}$ & $\begin{array}{l}\text { Calcium } \\
(\mathrm{mmol} / \mathrm{l})\end{array}$ & $\begin{array}{c}\text { Phosphate } \\
(\mathrm{mmol} / \mathrm{l})\end{array}$ & $\begin{array}{l}\text { Time } \\
\text { (days) }\end{array}$ & $\begin{array}{c}\text { Visual } \\
\text { observation }\end{array}$ & Absorbance & $\begin{array}{l}\text { Microscopic } \\
\text { observation }\end{array}$ & $\mathrm{pH}$ \\
\hline \multirow{8}{*}{$\mathrm{A} 1$} & \multirow[b]{2}{*}{ org 70} & \multirow{2}{*}{ org 50} & 0 & $x$ & $0.0015 \pm 0.0001$ & $x$ & $6.62 \pm 0.02$ \\
\hline & & & 31 & $x$ & $0.0079 \pm 0.0002$ & $x$ & $6.49 \pm 0.01$ \\
\hline & \multirow{2}{*}{ org 18} & \multirow{2}{*}{ inorg 18} & 0 & $x$ & $0.0003 \pm 0.0001$ & $\mathrm{x}$ & $6.01 \pm 0.00$ \\
\hline & & & 31 & V & $0.1773 \pm 0.009$ & V & $5.83 \pm 0.01$ \\
\hline & \multirow{2}{*}{ inorg 30} & \multirow{2}{*}{ org 30} & 0 & $x$ & $0.0052 \pm 0.0001$ & $x$ & $6.44 \pm 0.01$ \\
\hline & & & 31 & $x$ & $0.0004 \pm 0.0001$ & $x$ & $6.35 \pm 0.01$ \\
\hline & \multirow{2}{*}{ inorg 12} & \multirow{2}{*}{ inorg 15} & 0 & $x$ & $0.0010 \pm 0.0002$ & $x$ & $5.90 \pm 0.02$ \\
\hline & & & 31 & $x$ & $0.0021 \pm 0.0001$ & $x$ & $5.78 \pm 0.01$ \\
\hline \multirow{8}{*}{$\mathrm{A} 2$} & \multirow{2}{*}{ org 70} & \multirow{2}{*}{ org 50} & 0 & $x$ & $0.0080 \pm 0.0001$ & $x$ & $6.80 \pm 0.01$ \\
\hline & & & 31 & $x$ & $0.0033 \pm 0.0002$ & $x$ & $6.61 \pm 0.01$ \\
\hline & \multirow{2}{*}{ org 15} & \multirow{2}{*}{ inorg 15} & 0 & $x$ & $0.0009 \pm 0.0001$ & $x$ & $6.11 \pm 0.01$ \\
\hline & & & 31 & $x$ & $0.0023 \pm 0.0001$ & $x$ & $5.99 \pm 0.02$ \\
\hline & \multirow{2}{*}{ inorg 30} & \multirow{2}{*}{ org 30} & 0 & $x$ & $0.0008 \pm 0.0002$ & $x$ & $6.42 \pm 0.01$ \\
\hline & & & 31 & $x$ & $0.0079 \pm 0.0001$ & $x$ & $6.26 \pm 0.01$ \\
\hline & \multirow{2}{*}{ inorg 12} & \multirow{2}{*}{ inorg 15} & 0 & $\mathrm{x}$ & $0.0019 \pm 0.0001$ & $x$ & $6.06 \pm 0.00$ \\
\hline & & & 31 & V & $0.1789 \pm 0.007$ & V & $5.90 \pm 0.01$ \\
\hline \multirow{8}{*}{ A3 } & \multirow{2}{*}{ org 80} & \multirow{2}{*}{ org 60} & 0 & $x$ & $0.0096 \pm 0.0001$ & $x$ & $6.50 \pm 0.01$ \\
\hline & & & 31 & $x$ & $0.0062 \pm 0.0001$ & $x$ & $6.39 \pm 0.01$ \\
\hline & \multirow{2}{*}{ org 18} & \multirow{2}{*}{ inorg 18} & 0 & $\mathrm{x}$ & $0.0017 \pm 0.0002$ & $x$ & $6.00 \pm 0.01$ \\
\hline & & & 31 & V & $0.1109 \pm 0.006$ & V & $5.87 \pm 0.01$ \\
\hline & \multirow{2}{*}{ inorg 30} & \multirow{2}{*}{ org 30} & 0 & $x$ & $0.0037 \pm 0.0001$ & $\mathrm{x}$ & $6.25 \pm 0.02$ \\
\hline & & & 31 & $\mathrm{x}$ & $0.0008 \pm 0.0001$ & $\mathrm{x}$ & $6.12 \pm 0.02$ \\
\hline & \multirow{2}{*}{ inorg 15} & \multirow{2}{*}{ inorg 18} & 0 & $x$ & $0.0004 \pm 0.0001$ & $x$ & $5.91 \pm 0.02$ \\
\hline & & & 31 & V & $0.1003 \pm 0.008$ & V & $5.79 \pm 0.02$ \\
\hline \multirow[t]{2}{*}{ A4 } & \multirow{2}{*}{ org 90} & \multirow{2}{*}{ org 70} & 0 & $x$ & $0.0079 \pm 0.0001$ & $x$ & $6.94 \pm 0.01$ \\
\hline & & & 31 & $\mathrm{x}$ & $0.0060 \pm 0.0001$ & $x$ & $6.70 \pm 0.00$ \\
\hline
\end{tabular}




\begin{tabular}{|c|c|c|c|c|c|c|}
\hline \multirow{2}{*}{ org 18} & \multirow{2}{*}{ inorg 18} & 0 & $x$ & $0.0015 \pm 0.0002$ & $x$ & $5.89 \pm 0.01$ \\
\hline & & 31 & V & $0.2091 \pm 0.012$ & V & $5.56 \pm 0.01$ \\
\hline \multirow{2}{*}{ inorg 30} & \multirow{2}{*}{ org 30} & 0 & $x$ & $0.0059 \pm 0.0001$ & $x$ & $6.20 \pm 0.01$ \\
\hline & & 31 & $x$ & $0.0054 \pm 0.0002$ & $x$ & $6.19 \pm 0.02$ \\
\hline \multirow{2}{*}{ inorg 15} & \multirow{2}{*}{ inorg 18} & 0 & $x$ & $0.0005 \pm 0.0001$ & $x$ & $5.85 \pm 0.00$ \\
\hline & & 31 & $x$ & $0.0026 \pm 0.0001$ & $x$ & $5.69 \pm 0.01$ \\
\hline
\end{tabular}

$\mathrm{x}=$ no precipitation $\quad \mathrm{V}=$ precipitation

Table 4. Physicochemical properties of PN solution composition V $(n=9$; mean \pm SD; $p<0.05$, between 0 and 30 days +24 hours)

\begin{tabular}{|c|c|c|c|c|c|c|c|}
\hline $\begin{array}{c}\mathrm{PN} \\
\text { solution }\end{array}$ & $\begin{array}{l}\text { Calcium } \\
(\mathrm{mmol} / \mathrm{l})\end{array}$ & $\begin{array}{l}\text { Phosphate } \\
(\mathrm{mmol} / \mathrm{l})\end{array}$ & $\begin{array}{l}\text { Time } \\
\text { (days) }\end{array}$ & $\begin{array}{c}\text { Visual } \\
\text { observation }\end{array}$ & Absorbance & $\begin{array}{l}\text { Microscopic } \\
\text { observation }\end{array}$ & $\mathrm{pH}$ \\
\hline \multirow{8}{*}{ V1 } & \multirow{2}{*}{ org 70} & \multirow{2}{*}{ org 50} & 0 & $x$ & $0.0005 \pm 0.0001$ & $x$ & $6.70 \pm 0.01$ \\
\hline & & & 31 & $x$ & $0.0088 \pm 0.0002$ & $x$ & $6.55 \pm 0.01$ \\
\hline & \multirow{2}{*}{ org 18} & \multirow{2}{*}{ inorg 18} & 0 & $\mathrm{x}$ & $0.0004 \pm 0.0001$ & $\mathrm{x}$ & $6.09 \pm 0.01$ \\
\hline & & & 31 & $x$ & $0.0006 \pm 0.0001$ & $x$ & $5.83 \pm 0.01$ \\
\hline & \multirow{2}{*}{ inorg 30} & \multirow{2}{*}{ org 30} & 0 & $x$ & $0.0010 \pm 0.0002$ & $x$ & $6.54 \pm 0.01$ \\
\hline & & & 31 & $x$ & $0.0013 \pm 0.0001$ & $x$ & $6.31 \pm 0.01$ \\
\hline & \multirow{2}{*}{ inorg 15} & \multirow{2}{*}{ inorg 18} & 0 & $x$ & $0.0071 \pm 0.0001$ & $x$ & $5.99 \pm 0.01$ \\
\hline & & & 31 & V & $0.2132 \pm 0.011$ & V & $5.78 \pm 0.01$ \\
\hline \multirow{8}{*}{ V2 } & \multirow{2}{*}{ org 80} & \multirow{2}{*}{ org 60} & 0 & $\mathrm{x}$ & $0.0062 \pm 0.0001$ & $\mathrm{x}$ & $6.57 \pm 0.02$ \\
\hline & & & 31 & $x$ & $0.0004 \pm 0.0001$ & $x$ & $6.49 \pm 0.01$ \\
\hline & \multirow{2}{*}{ org 18} & \multirow{2}{*}{ inorg 18} & 0 & $x$ & $0.0006 \pm 0.0002$ & $x$ & $5.95 \pm 0.01$ \\
\hline & & & 31 & $\mathrm{~V}$ & $0.1891 \pm 0.009$ & V & $5.80 \pm 0.01$ \\
\hline & \multirow{2}{*}{ inorg 30} & \multirow{2}{*}{ org 30} & 0 & $x$ & $0.0015 \pm 0.0001$ & $x$ & $6.30 \pm 0.01$ \\
\hline & & & 31 & $x$ & $0.0029 \pm 0.0001$ & $x$ & $6.05 \pm 0.01$ \\
\hline & \multirow{2}{*}{ inorg 15} & \multirow{2}{*}{ inorg 18} & 0 & $x$ & $0.0022 \pm 0.0002$ & $\mathrm{x}$ & $5.64 \pm 0.00$ \\
\hline & & & 31 & $\mathrm{x}$ & $0.0099 \pm 0.0003$ & $\mathrm{x}$ & $5.56 \pm 0.01$ \\
\hline \multirow{10}{*}{ V3 } & \multirow{2}{*}{ org 80} & \multirow{2}{*}{ org 60} & 0 & $x$ & $0.0028 \pm 0.0002$ & $x$ & $6.35 \pm 0.01$ \\
\hline & & & 31 & $x$ & $0.0031 \pm 0.0001$ & $x$ & $6.21 \pm 0.01$ \\
\hline & \multirow{2}{*}{ org 18} & \multirow{2}{*}{ inorg 18} & 0 & $x$ & $0.0015 \pm 0.0001$ & $x$ & $5.68 \pm 0.01$ \\
\hline & & & 31 & $x$ & $0.0007 \pm 0.0001$ & $x$ & $5.60 \pm 0.01$ \\
\hline & \multirow{2}{*}{ inorg 30} & \multirow{2}{*}{ org 30} & 0 & $x$ & $0.0016 \pm 0.0001$ & $\mathrm{x}$ & $6.05 \pm 0.02$ \\
\hline & & & 31 & $x$ & $0.0055 \pm 0.0002$ & $x$ & $5.90 \pm 0.02$ \\
\hline & \multirow{2}{*}{ inorg 15} & \multirow{2}{*}{ inorg 18} & 0 & $x$ & $0.0002 \pm 0.0001$ & $x$ & $5.58 \pm 0.02$ \\
\hline & & & 31 & V & $0.1193 \pm 0.010$ & V & $5.47 \pm 0.02$ \\
\hline & \multirow{2}{*}{ org 90} & \multirow{2}{*}{ org 70} & 0 & $x$ & $0.0072 \pm 0.0003$ & $x$ & $6.80 \pm 0.01$ \\
\hline & & & 31 & $\mathrm{x}$ & $0.0060 \pm 0.0001$ & $\mathrm{x}$ & $6.62 \pm 0.01$ \\
\hline
\end{tabular}




\begin{tabular}{|c|c|c|c|c|c|c|c|}
\hline \multirow{6}{*}{ V4 } & \multirow{2}{*}{ org 18} & \multirow{2}{*}{ inorg 18} & 0 & $x$ & $0.0003 \pm 0.0001$ & $x$ & $5.79 \pm 0.00$ \\
\hline & & & 31 & $x$ & $0.0013 \pm 0.0001$ & $x$ & $5.62 \pm 0.01$ \\
\hline & \multirow{2}{*}{ inorg 30} & \multirow{2}{*}{ org 30} & 0 & $x$ & $0.0082 \pm 0.0002$ & $x$ & $6.19 \pm 0.01$ \\
\hline & & & 31 & $x$ & $0.0052 \pm 0.0001$ & $x$ & $6.11 \pm 0.01$ \\
\hline & \multirow{2}{*}{ inorg 15} & \multirow{2}{*}{ inorg 18} & 0 & $x$ & $0.0022 \pm 0.0001$ & $x$ & $5.64 \pm 0.01$ \\
\hline & & & 31 & $x$ & $0.0006 \pm 0.0001$ & $x$ & $5.50 \pm 0.02$ \\
\hline
\end{tabular}

\subsection{Microscopic Observation}

Microscopic observations of the PN solutions confirmed the visual observations (Table 2-4). The amorphous, spongy precipitate, forming larger agglomerates was present in PN solutions with organic calcium and phosphate ions at concentrations above the therapeutic range (Figure 5). Crystal precipitation in the range of 50 to $100 \mu \mathrm{m}$ in size was visible in PN solutions containing inorganic ions (Table 2-4). The crystals appeared individually in the field of view or formed compact structures (Figure 6).
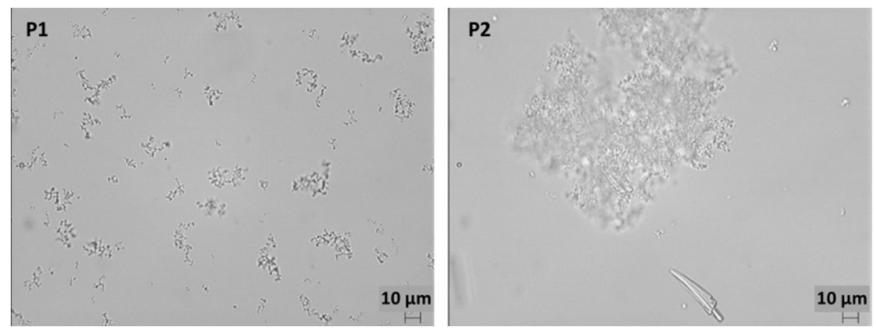

Fig. 5. Amorphous precipitate of organic calcium $(90 \mathrm{mmol} / \mathrm{L})$ and organic phosphate $(70 \mathrm{mmol} / \mathrm{L})$ in PN solutions P1 and P2.
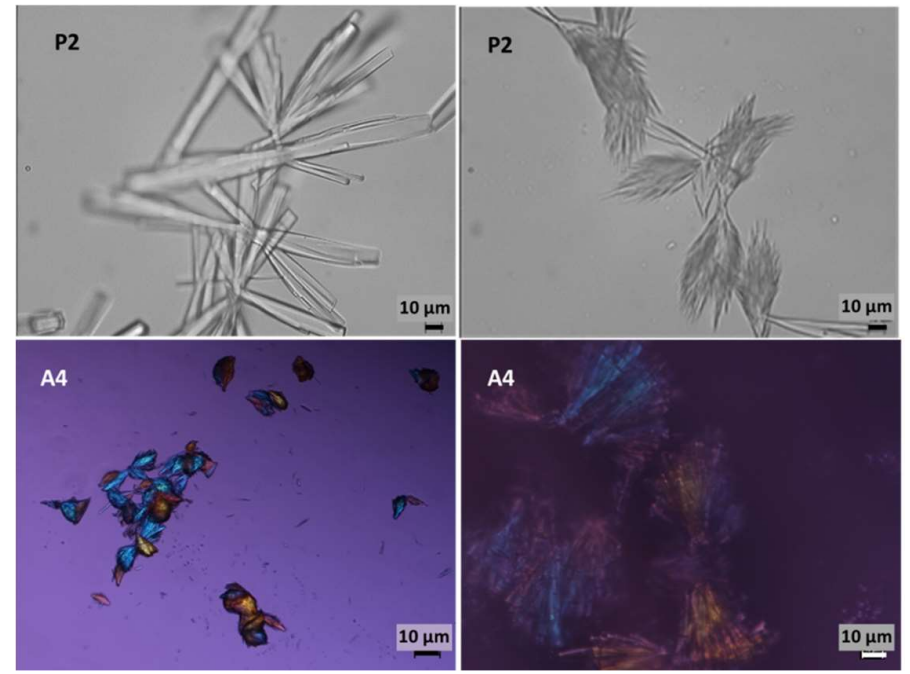

Fig. 6. Crystal precipitation of inorganic calcium and inorganic phosphate in PN solutions P2 and A4.

\section{3. pH Measurement}


The $\mathrm{pH}$ of the PN solutions ranged from 5.23 to 7.18 (Table 2). Higher concentrations of organic calcium and phosphate ions were characterised by the highest $\mathrm{pH}$ values. There was no relationship between the presence of precipitate and change in $\mathrm{pH}$ values. A statistically significant decrease $(\mathrm{p}<0.05)$ from the baseline $(\mathrm{t}=0)$ of each composition of $\mathrm{PN}$ solution to 30 days $+24 \mathrm{~h}$ was observed (Figure 7, Table 2-4), which possibly resulted from the removal of HPO42- (monohydrogen phosphate) by the reaction with calcium or the unmeasured degradation of amino acids and glucose.

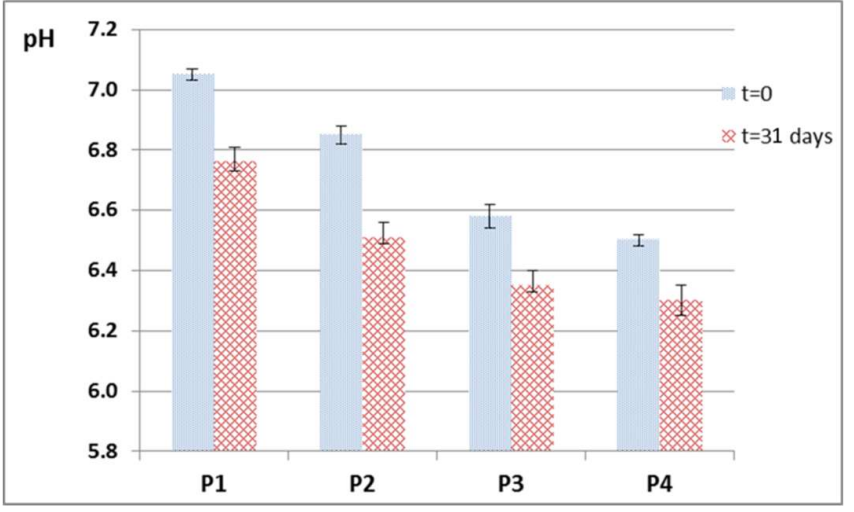

Fig. 7. $\mathrm{pH}$ values of $\mathrm{PN}$ solutions (compositions $\mathrm{P}$ ) with organic calcium $(50 \mathrm{mmol} / \mathrm{L})$ and organic phosphate $(30 \mathrm{mmol} / \mathrm{L})$ - the influence of storage.

\subsection{Absorbance Measurement}

PN solutions with calcium and phosphate with no visual changes as well with turbidity were examined in the visible light range. The absorbance at $600 \mathrm{~nm}$ was in the range of $0.0001-0.0120$ (Table 2-4) for most of the samples. The absorbance above 0.1000 was measured for turbidity samples with precipitate (Table 2-4).

\subsection{Risk curves}

Using spectrophotometric measurement as well as visual and microscopic observation, maximum, safe for therapeutic use, calcium and phosphate ions concentrations were determined to ensure no precipitation at $\mathrm{t}=24 \mathrm{~h}\left(37^{\circ} \mathrm{C}\right)$. Figures $2-4$ show the risk curves of precipitation in the tested PN solutions, depending on the type and concentration of calcium and phosphate ions. The safe zone (green) includes the range of ion concentrations at which precipitation did not occur within 24 hours at $37^{\circ} \mathrm{C}$ after combining. A high risk zone (orange) means a concentration range that was not measurable. There were different limit concentrations at which no precipitation occurred after 24 hours. Limit concentrations depended on the composition of the aqueous phase. Further increases in ion concentration caused precipitation (red zone).

\section{Discussion}

Maximising calcium and phosphates ions concentrations without increasing the risk of precipitation is a challenge for manufacturers of PN solutions, particularly in the neonatology. Indeed, premature neonates have very high calcium $(\mathrm{Ca})$ and phosphate (PO4) requirements for bone mineralisation and are often have restricted fluid intake and many parenteral solution are compounded close to saturation point [9]. This limits the practitioner's ability to adequately supply the required amounts of calcium and phosphate especially in small patients. Furthermore, varying the concentration of 
other components of the parenteral solution such as amino acids, glucose, electrolytes and lipids can alter the saturation point, potentially increasing the probability of precipitation.

The nature of calcium and phosphate salts directly influences the solubility of calcium phosphate. Given the differences in dissociation characteristics, the relative concentrations of calcium or phosphate available for precipitation are higher when inorganic salts are used [1]. The general suggestion when compounding parenteral solutions, especially for premature infants, is to use organic phosphate (glycerophosphate) together with organic calcium salts (calcium gluconate) as an uncomplicated and safe way to simultaneously administer high amounts of calcium and phosphate in TPN solutions [3]. Organic calcium salts such as calcium gluconate (Ca-glu) are widely used but are temporarily unavailable in Poland due to drug shortages. The availability of organic phosphate salts (sodium glucose-1-phosphate, G1P as well as sodium glycerophosphate, NaGP) is limited too because they are not registered and approved for use in every country [13,14]. In hospital practice it is very valuable to know if electrolyte replacement will be safe for patients. Due to the various combinations of composition of parenteral admixture solution for small patients and variety of calcium and phosphate salts available it is very difficult to establish safe limits of calcium and phosphate concentrations.

In this study, the experimental information provided is useful for optimising the hospital compounding of PN solutions containing calcium and phosphate ions. The solubility of calcium phosphate depends on several variables such as $\mathrm{pH}$ or the concentration of amino acids and glucose, however, the most relevant factor is the nature of the salt (organic or inorganic). It is impossible to establish rules to predict the precipitation and each composition should be studied individually. Our study was performed in various conditions initially from those particularly prone to precipitation (low concentration of amino acids and glucose - composition "1"). Our data provide evidence that even in these worst-case conditions, a wide range (above the therapeutic range) of PN solutions with up to $80 \mathrm{mmol} / \mathrm{L}$ of organic calcium ions and $60 \mathrm{mmol} / \mathrm{L}$ of organic phosphate ions are stable for 30 days at $4^{\circ} \mathrm{C}$ and following $24 \mathrm{~h}$ at $37^{\circ} \mathrm{C}$. Consequently, the limits included in our prescribing software were increased, allowing the prescription of compositions of PN solutions with calcium and phosphate ions up to $80 \mathrm{mmol} / \mathrm{L}$ and $60 \mathrm{mmol} / \mathrm{L}$, respectively. This study helps physicians in daily practice who are no longer limited in their prescriptions of calcium and phosphate ions. A second useful aspect of this study is economically advantageous - giving curves within safe zone allows the use of inorganic salts which are much cheaper than organic calcium and phosphate salts. The third advantage is that it overcomes the shortage of organic salts which very often occurs in hospital practice and prevents the safe administration of PN solutions.

Most investigators identify calcium phosphate precipitation by visual inspection. However, visual inspection can sometimes be ineffective in preventing precipitate in parenteral solution, especially for all-in-one admixtures in which lipid emulsions are present or when calcium phosphate precipitation is not enough to be visible (at the beginning of this process). The second limitation is the fact that visual inspection is very subjective and does not allow distinguishing calcium phosphate precipitation from other precipitation. Other analytical methods should be applied to ensure safe therapy for patients.

Light microscopy can be used to detect the particle counts in solutions as well as on filter surfaces after filtration of the PN solution [7]. The isolation of particles by filtration of the PN solution followed by microscopic observation of the filter surfaces is time-consuming but is a relatively sensitive method. It should be kept in mind that it depends on particle suspension before 
filtration and it also does not differentiate between calcium phosphate precipitation and other particles present in the mixture.

The absorbance from UV spectrophotometer to determine the presence of precipitation was also involved [8]. An absorbance greater than 0.015 at $600 \mathrm{~nm}$ was considered evidence of precipitation [15].

The maximum concentrations of calcium and phosphate ions without precipitation up to 24 hours at $37^{\circ} \mathrm{C}$ were established for each tested composition of PN solution. Our results guarantee safe parenteral therapy for neonates. The focus was on salt combinations containing at least one type of ion in an inorganic form. In addition, the minimum concentrations of organic calcium and phosphate salts which cause precipitation were studied. For this case, the salts were tested in ranges much higher than the therapeutic values. The composition of PN solutions were the ones most commonly used in clinical practice. Three different paediatric amino acids solutions (Primene, Aminoven, Vaminolact) were used. The PN solutions varied in their glucose and amino acid concentrations. In addition, the studied solutions were stored for up to 30 days to check the effect of time on their properties. The results confirmed the protective effect of amino acids on PN solutions with a higher concentration of amino acids, no precipitation was observed despite the high concentrations of calcium and phosphate ions (Table 2-4). In PN solutions with a lower concentration of amino acids, the presence of a precipitate in the same ion range was noted (P1 and P2 vs P3 and P4).

Inorganic calcium and phosphate salts were characterised by a higher risk of precipitation than organic salts (Figure 2-4). For a combination of inorganic calcium salts and organic phosphate salts it was possible to use higher concentrations than in the reverse case (Figure 2-4). The generally accepted principle regarding organic calcium and phosphate ions is that they can be combined without restrictions because no precipitation will occur. It was shown that there is a risk of precipitation, nevertheless, it occurs outside the therapeutic range of concentrations (calcium ions 50 $\mathrm{mmol} / \mathrm{L}$, phosphate ions $30 \mathrm{mmol} / \mathrm{L}$ ) (Figure 2-4).

The $\mathrm{pH}$ values increased with increasing concentration of organic ions (Table 2-4). However, the studies showed a significant decrease in the $\mathrm{pH}$ value with the storage time for aqueous phases with organic calcium and phosphate ions (Table 2-4). However, the presence of precipitation has no influence on this parameter.

Only a combination of various technical and time-consuming methods used by laboratories could ensure detection of precipitation in a PN solution. However, in hospital practice, using a relatively simple method such as visual examination, with appropriate illumination and background, should always be applied as it as reliable as a general screening method.

\section{Conclusion}

The maximum safe combination of calcium and phosphate for each investigated composition of PN solution, even in the worst-case situations, was proposed. This work is valuable in daily practice as it allows an increase in the limits of calcium and phosphate in PN solutions for infants. This study provides valuable data on the compatibility of inorganic and organic calcium salts with inorganic and organic phosphate salts in various combinations in PN solutions compounded for neonates. This data adds to the literature information which may be used to evaluate different options for administering calcium and phosphate in neonatal PN solutions. This may be valuable information 
during times of a shortage of PN organic additives of calcium and phosphate. It should be pointed out that any change in the composition of PN solutions needs new analysis.

Funding: This research received no external funding.

Acknowledgements: I would like to thank the pharmacists from Hospital Pharmacy at the Stanley Dudrick's Memorial Hospital in Skawina for compounding the PN solutions.

Conflicts of Interest: The author declares no conflict of interest.

\section{References}

1. Bouchoud, L.; Fonzo-Christe, C.; Sadeghipour, F.; Bonnabry, P. Maximizing calcium and phosphate content in neonatal parenteral nutrition solutions using organic calcium and phosphate salts. J. Parenter. Enter. Nutr. 2010, 34, 542-545, doi:10.1177/0148607110374615.

2. Anderson, C.; MacKay, M. Physical Compatibility of Calcium Chloride and Sodium Glycerophosphate in Pediatric Parenteral Nutrition Solutions. J. Parenter. Enter. Nutr. 2016, 40, 1166-1169, doi:10.1177/0148607115592673.

3. Chaieb D., S.; Chaumeil, J. C.; Jebnoun, S.; Khrouf, N.; Hedhili, A.; Sfar, S. Effect of the intravenous lipid emulsions on the availability of calcium when using organic phosphate in TPN admixtures. Pharm. Res. 2008, 25, 2545-2554, doi:10.1007/s11095-008-9671-7.

4. Popinska, K.; Ksiazyk, J.; Friedman-Gruszczynska, J.; Nowicka, E.; Migdal, A.; Pietraszek, E. Aluminum concentration in serum of children on long-term parenteral nutrition and in parenteral nutrition solution components. e-SPEN 2010, 5, e18-e20, doi:10.1016/j.eclnm.2009.10.010.

5. Poole, R. L.; Pieroni, K. P.; Gaskari, S.; Dixon, T.; Kerner, J. A. Aluminum exposure in neonatal patients using the least contaminated parenteral nutrition solution products. Nutrients 2012, 4, 1566-1574, doi:10.3390/nu4111566.

6. Migaki, E. A.; Melhart, B. J.; Dewar, C. J.; Huston, R. K. Calcium chloride and sodium phosphate in neonatal parenteral nutrition containing TrophAmine: Precipitation studies and aluminum content. J. Parenter. Enter. Nutr. 2012, 36, 470-475, doi:10.1177/0148607111420154.

7. Allwood, M. C.; Kearney, M. C. J. Compatibility and stability of additives in parenteral nutrition admixtures. Nutrition 1998, 14, 697-706, doi:10.1016/S0899-9007(98)00063-X.

8. Thowladda, N.; Siritientong, T. Compatibility of calcium and sodium glycerophosphate in parenteral nutrition solutions. Thai J. Pharm. Sci. 2016, 40, 176-179.

9. MacKay, M.; Anderson, C. Physical Compatibility of Sodium Glycerophosphate and Calcium Gluconate in Pediatric Parenteral Nutrition Solutions. J. Parenter. Enter. Nutr. 2015, 39, 725728, doi:10.1177/0148607114528982.

10. Watrobska-Swietlikowska, D.; MacLoughlin, R.; Szlagatys-Sidorkiewicz, A. The presence of inorganic calcium in pediatric parenteral admixtures. Nutr Hosp 2016, 35, 11-18, doi:10.3305/nh.2013.28.sup4.6783.

11. Ribeiro, D. D. O.; Lobo, B. W.; Volpato, N. M.; Da Veiga, V. F.; Cabral, L. M.; De Sousa, V. P. Influence of the calcium concentration in the presence of organic phosphorus on the physicochemical compatibility and stability of all-in-one admixtures for neonatal use. Nutr. J. 
2009, 8, 1-13, doi:10.1186/1475-2891-8-51.

12. Newton, D. W.; Driscoll, D. F. Calcium and phosphate compatibility: Revisited again. Am. J. Heal. Pharm. 2008, 65, 73-80, doi:10.2146/ajhp070138.

13. Huston, R. K.; Christensen, J. M.; Alsharhani, S. M.; Mohamed, S. M.; Heisel, C. F. Calcium chloride and calcium gluconate in neonatal parenteral nutrition solutions without cysteine: Compatibility studies using laser light obscuration methodology. Nutrients 2018, 10, doi:10.3390/nu10020208.

14. Joy, J.; Silvestri, A. P.; Franke, R.; Bistrian, B. R.; Nehne, J.; Newton, D. W.; Driscoll, D. F. Calcium and phosphate compatibility in low-osmolarity parenteral nutrition admixtures intended for peripheral vein administration. J. Parenter. Enter. Nutr. 2010, 34, 46-54, doi:10.1177/0148607109338216.

15. Niermeyer, S.; Thieme, R.; Givan, C.; Thibodeau, S.; Quissell, B. J. Optimized calcium/phosphorus solubility in a parenteral nutrition solution containing dicarboxylic amino acids and cysteine. J. Am. Coll. Nutr. 1986, 5, 459-466, doi:10.1080/07315724.1986.10720149. 